\title{
The Crystal Structure of the Plasmodium falciparum PdxK Provides an Experimental Model for Pro-Drug Activation
}

\author{
Kai Gao $\left.{ }^{1}{ }^{(}\right)$, Wenjia Wang ${ }^{1}\left(\mathbb{D}\right.$, Thales Kronenberger ${ }^{2,3}{ }^{\oplus}$, Carsten Wrenger ${ }^{3}$ and \\ Matthew R. Groves ${ }^{1, *}$ \\ 1 Drug Design XB20, Groningen Research Institute of Pharmacy, University of Groningen, 9700 AD Groningen, \\ The Netherlands; k.gao@rug.nl (K.G.); w.wang@rug.nl (W.W.) \\ 2 Department of Medical Oncology \& Pneumology, Internal Medicine VIII, University Hospital Tübingen, \\ 72076 Tübingen, Germany; kronenberger7@gmail.com \\ 3 Unit for Drug Discovery, Department of Parasitology, Institute of Biomedical Sciences, University of São \\ Paulo, 05508-000 São Paulo, Brazil; cwrenger@icb.usp.br \\ * Correspondence: m.r.groves@rug.nl; Tel.: +31-(0)50-363-3305
}

Received: 2 August 2019; Accepted: 11 October 2019; Published: 17 October 2019

\begin{abstract}
Pyridoxine/pyridoxal kinase (PdxK), belongs to the ribokinase family and is involved in the vitamin B6 salvage pathway by phosphorylating 5-pyridoxal (PL) into an active form. In the human malaria parasite, Plasmodium falciparum, $\mathrm{Pf} \mathrm{PdxK}$ functions to salvage vitamin B6 from both itself and its host. Here, we report the crystal structure of $P f \mathrm{PdxK}$ from P. falciparum in complex with a non-hydrolyzable ATP analog (AMP-PNP) and PL. As expected, the fold is retained and both AMP-PNP and PL occupy the same binding sites when compared to the human ortholog. However, our model allows us to identify a FIxxIIxL motif at the $\mathrm{C}$ terminus of the disordered repeat motif $(\mathrm{XNXH})_{\mathrm{m}}$ that is implicated in binding the WD40 domain and may provide temporal control of $P f \mathrm{PdxK}$ through an interaction with a E3 ligase complex. Furthermore, molecular docking approaches based on our model allow us to explain differential $P f \mathrm{PdxK}$ phosphorylation and activation of a novel class of potent antimalarials (PT3, PT5 and PHME), providing a basis for further development of these compounds. Finally, the structure of $P f \mathrm{PdxK}$ provides a high-quality model for a better understanding of vitamin B6 synthesis and salvage in the parasite.
\end{abstract}

Keywords: PfPdxK; motif; PT3; PT5; PHME

\section{Introduction}

Pyridoxal 5'-phosphate (PLP) is the active form of vitamin B6, a cofactor for many enzymes involved in amino acid and sugar metabolism [1]. Many species possess both a de novo PLP synthetic pathway, as well as a salvage pathway, to take up vitamin B6 from nutrients. In the presence of ATP and $\mathrm{Mg}^{2+}$, precursors of an active form of vitamin B6 (pyridoxine (PN), pyridoxal (PL), and pyridoxamine $(\mathrm{PM})$ ) are phosphorylated by PL kinase (PdxK) through transfer of a phosphate group from ATP to the 5'-hydroxyl group of PL [2,3]. PL kinase has been studied and purified from bacterial, plants, and mammalian sources and most organisms contain a single PL kinase, coded for by the PdxK gene $[4,5]$. The crystal structures of sheep brain and human PdxK have been previously published [6,7]. Additionally, E. coli PdxK structures have been determined both as native and binary complexes (with either Mg:ATP and PL) [8]. Despite a low sequence identity among these organisms, they share a highly similar structure and fold [9]. As vitamin salvage has long been thought to be a potential area for the development of novel antimalarials, the PdxK from P. falciparum has been studied by electron microscopy [10], and we have previously reported the crystallisation of PfPdxK [11]. 
Unlike mammalian cells, P. falciparum has a functional vitamin B6 de novo biosynthetic pathway [12], which has been previously validated as a drug target [13]. The parasite also possesses an interconversion pathway reliant upon $P f \mathrm{PdxK}$, which has been examined for pro-drug discovery [14]. A strategy of impairing the growth of $P$. falciparum was suggested, in which pro-drugs are phosphorylated within the parasite to generate pyridoxyl-amino acid adducts that block PLP-dependent enzymes, inhibiting proliferation [15]. Three novel non-phosphorylated pyridoxyl-adducts compounds (PT3, PT5 and PHME) were tested in an anti-plasmodial assay, with results showing that PT3 can inhibit the proliferation of P. falciparum with an $\mathrm{IC}_{50}$ of $14 \mu \mathrm{M}$ [15]. Further development of this strategy requires high-resolution models of both the human and plasmodial PdxK, in order to guide improvements in specificity.

Pf PdxK is also rather unique amongst PdxKs, as malarial sp. PdxKs possess a highly degenerate internal motif $(\mathrm{XNXH})_{\mathrm{m}}$ that shares similarities with motifs involved in targeting protein for degradation, thereby potentially providing temporal control of $\mathrm{PdxK}$ activity. This type of degenerate motif is common within the malarial genome [16] and little research has been performed on any advantage such degenerate motifs may convey to the parasite. Interestingly, while this degenerate loop was predicted to be non-structured, a second accompanying motif (FxIxxIL) found that the N-terminal to $(\mathrm{XNXH})_{\mathrm{m}}$ may provide some insight into the molecular interactions between PfPdxK (and other degenerate repeat containing proteins) and the molecules(s) likely to govern their degradation.

In this manuscript, we report the crystal structure of the PdxK:Mg:AMP-PNP:PL complex from P. falciparum determined by X-ray diffraction at a resolution of $2.15 \AA$. We show, as expected, that the overall fold is highly conserved with other members of the PdxK family and that the $(\mathrm{XNXH})_{\mathrm{m}}$ motif is disordered. However, the (FIxxIIxL) motif is structured and provides a surface-exposed epitope that shares similarities with the EH1 motif that plays a role in recruiting WD40 domains. Molecular modelling also demonstrated the molecular rational for the improved behavior of PTME and PT5 over that of PT3.

\section{Materials and Methods}

\subsection{Purification and Crystallization}

The cloning, expression, buffer optimization and crystallization procedures of native PfPdxK have been described previously [11]. Briefly, $P f \mathrm{PdxK}$ was recombinantly expressed in E. coli Rosetta 2 (DE3) cells, followed by propagation in LB medium supplemented with $4 \mathrm{mM} \mathrm{MgCl} 2$ at $37^{\circ} \mathrm{C}$, after reaching the $\mathrm{OD}_{600}$ at 0.6 , anhydrotetracycline was added for induction of protein expression and the culture was further incubated at $18{ }^{\circ} \mathrm{C}$ overnight until the pellets were harvested. Initial purification was performed using the affinity chromatography column, HisTrap FF (GE Healthcare). The protein was eluted with loading buffer supplemented with $300 \mathrm{mM}$ imidazole, then the protein was pooled, concentrated and purified by size exclusion chromatography using column HiLoad 16/60 Superdex 75 (GE Healthcare). Protein purity was assessed by SDS-PAGE and apo crystals appeared in hanging-drop experiments performed at $20^{\circ} \mathrm{C}$ using a crystallization buffer containing $0.1 \mathrm{M}$ HEPES, pH 7.75, $0.2 \mathrm{M} \mathrm{CaCl}_{2}, 31 \%(v / v)$ PEG 400, 5\% (v/v) glycerol. AMP-PNP and PL (each $5 \mathrm{mM}$ ) were added into the drops containing apo PfPdxK crystals for soaking. The resulting crystals were then flash-cooled in liquid nitrogen with an additional $25 \%(\mathrm{v} / \mathrm{v})$ glycerol as a cryo-protectant. All chemicals were purchased through VWR, Amsterdam, The Netherlands.

\subsection{Data Collection and Structure Determination}

X-ray diffraction data were collected at EMBL Hamburg and P11, the PETRA III synchrotron facility, Hamburg, Germany. Diffraction images were processed with XDS [17], scaled and merged with Aimless [18]. While the crystals displayed a variation in the recorded diffraction resolution from $3.4 \AA$ to $2.1 \AA$, all PfPdxK complex crystals were processed in space group P1211. An overview of the data collection and refinement statistics can be found in Table 1. The structures of the PfPdxK/AMP-PNP complex were solved by molecular replacement with PHASER [19] using the coordinates of PdxK from sheep brain as a search model (PDB entry 1RFU [6]). The model was further improved by iterative 
cycles of manual rebuilding via Coot [20] and refinement using Refmac5 [21] to improve the electron density map. All refinement steps were carried out automatically, using software default values and applying non-crystallographic restraints.

Table 1. Data collection and refinement statistics.

\begin{tabular}{|c|c|}
\hline Data Set & PfPdxK-AMP-PNP-PL \\
\hline Wavelength & $0.97 \AA(12.398 \mathrm{keV})$ \\
\hline Resolution range & $19.33-2.15(2.227-2.15)$ \\
\hline Space group & P 1211 \\
\hline Unit cell & 52.70362 .00493 .7129094 .98890 \\
\hline Total reflections & $81052(8023)$ \\
\hline Unique reflections & $32284(3159)$ \\
\hline Multiplicity & $2.5(2.6)$ \\
\hline Completeness (\%) & $97.87(97.17)$ \\
\hline Mean I/sigma(I) & $15.57(2.69)$ \\
\hline Wilson B-factor & 39.31 \\
\hline R-merge & $3.7(38.61)$ \\
\hline R-meas & $4.7(49.31)$ \\
\hline CC $1 / 2$ & $0.99(0.88)$ \\
\hline $\mathrm{CC}^{*}$ & $1(0.967)$ \\
\hline Reflections used in refinement & 32276 (3159) \\
\hline Reflections used for R-free & $1627(165)$ \\
\hline R-work & $0.2094(0.3002)$ \\
\hline R-free & $0.2718(0.3691)$ \\
\hline Number of non-hydrogen atoms & 4885 \\
\hline macromolecules & 4727 \\
\hline ligands & 89 \\
\hline solvent & 69 \\
\hline Protein residues & 591 \\
\hline RMS(bonds) & 0.008 \\
\hline RMS(angles) & 0.95 \\
\hline Ramachandran favored (\%) & 95.62 \\
\hline Ramachandran allowed (\%) & 4.20 \\
\hline Ramachandran outliers (\%) & 0.18 \\
\hline Rotamer outliers (\%) & 0.00 \\
\hline Clashscore & 8.69 \\
\hline Average B-factor & 59.75 \\
\hline macromolecules & 59.58 \\
\hline ligands & 73.01 \\
\hline solvent & 54.41 \\
\hline
\end{tabular}

The stereochemical properties of the final models were analyzed with the Molprobity server (http://molprobity.biochem.duke.edu). At least $95 \%$ of the residues are found in the favoured region of the Ramachandran plot. Protein-PL interactions were analyzed with the scorpion server (http://www.desertsci.com/). Structural alignments/analysis and all structural figures were prepared with PyMOL (Graphic System, Schrodinger, LLC). The structure has been deposited with the PDB under the accession code $6 \mathrm{SU} 9$.

\subsection{Molecular Docking}

Small-molecule docking of PfPdxK with PT3, PT5 and PMME were performed by rigid-docking using Swiss dock [22] (http://www.swissdock.ch/docking), based on EADock. The coordinate file PfPdxK was edited to remove all water molecules and PL, with AMP-PNP maintained in position to provide a realistic model of ATP binding. Compounds were optimized using MM2 via Chemdraw. The ligand-binding site was constrained in a $10 \times 10 \times 10 \AA^{3}$ cube with the protein at the center. Flexible side chains were not allowed. All rotatable single bonds were allowed to rotate within the ligand. Docking results were screened by Chimera.

\section{Results}

\subsection{PfPdxK Adopts a Highly Similar Structure to that Seen in Different Species}

Multiple sequences and structures alignment were generated from T-Coffee [23] and ENDscript 2.0 [24]. We selected three published crystals structures from human, sheep brain and E. coli as reference 
PdxK structures. PfPdxK consists of 493 amino acids, of which, only 54\% overlaps with the sequences of other species (Figure 1). Amongst this $54 \%$, only $30 \%$ of the sequence was conserved. The insertion from His107 to Tyr303 consists of repeated-motif MNXH or TNXH peptides, with no known homology within the PDB (Figure 1). Sequencing of the expression plasmid confirm the completeness of the protein, with no detectable degradation visible [11]. Unfortunately, no electron density was visible for the degenerate region and there is likely no defined structure of the repeated motif. Based on our data, it seems that it does not influence the structure of $P f \mathrm{PdxK}$.

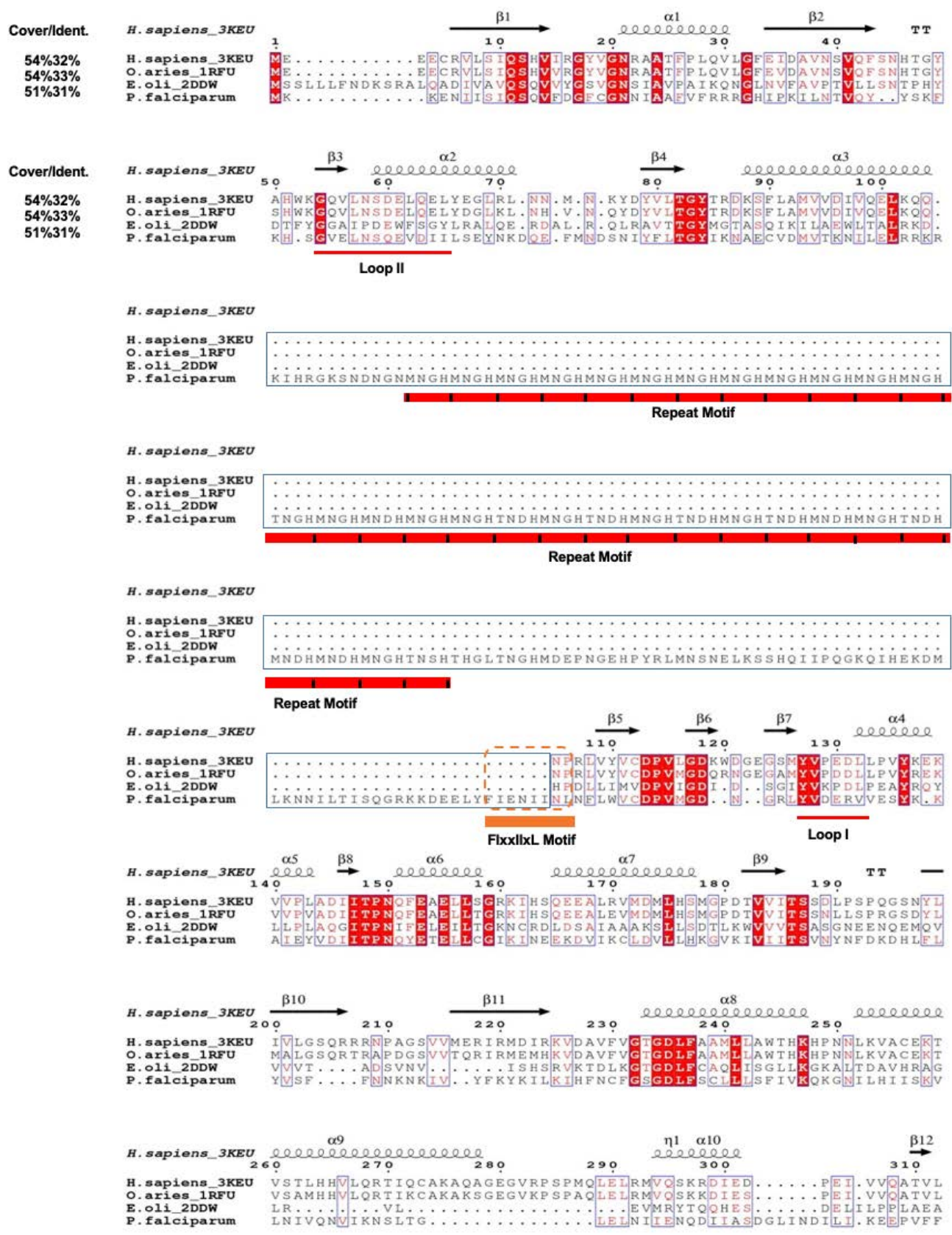

Figure 1. Multiple sequence alignment of PfPdxK and the indicated homologs available within the PDB were generated using BLAST, T-Coffee and ENDscript 2.0. PfPdxK exhibits a sequence identity of $30 \%$ over $54 \%$ of its sequence, with the remainder of the sequence present as an extended, high-degenerate insert consisting of multiple MNXH or TNXH repeats. Such repeats are frequently observed within the malarial genome (Figure S1 in Supplementary Materials), the Eh1-like motif FIxxIIxL was labeled in orange after the repeat motifs. 
We performed a structural alignment of $P f \mathrm{PdxK}$ against the human PdxK structure to uncover any conformational changes of $P f \operatorname{PdxK}$ that may be driven by the internal repeat elements (Figure 2). As $\mathrm{PdxK}$ activity is retained in vivo, $P f \mathrm{PdxK}$ unsurprisingly followed the basic structural organization of PdxK proteins, with the overall structure showing the same conformation as that typically seen within the ribokinase superfamily: each monomer consists of seven core $\beta$ strands $(\beta 1-\beta 7)$ with five parallel and one antiparallel strand, surrounded by nine $\alpha$-helices $(\alpha 1-\alpha 9)$ as indicated, with $\alpha 2$ slightly offset against $\alpha 3$. The active site is present as a shallow groove, flanked by conserved loop 1 , with which ATP or AMP-PNP predominantly interacts. The 5-pyridoxal substrate binding site is equally conserved and almost completely buried in all structures, strongly suggesting a degree of rearrangement will be required during substrate loading and product dissociation [6].

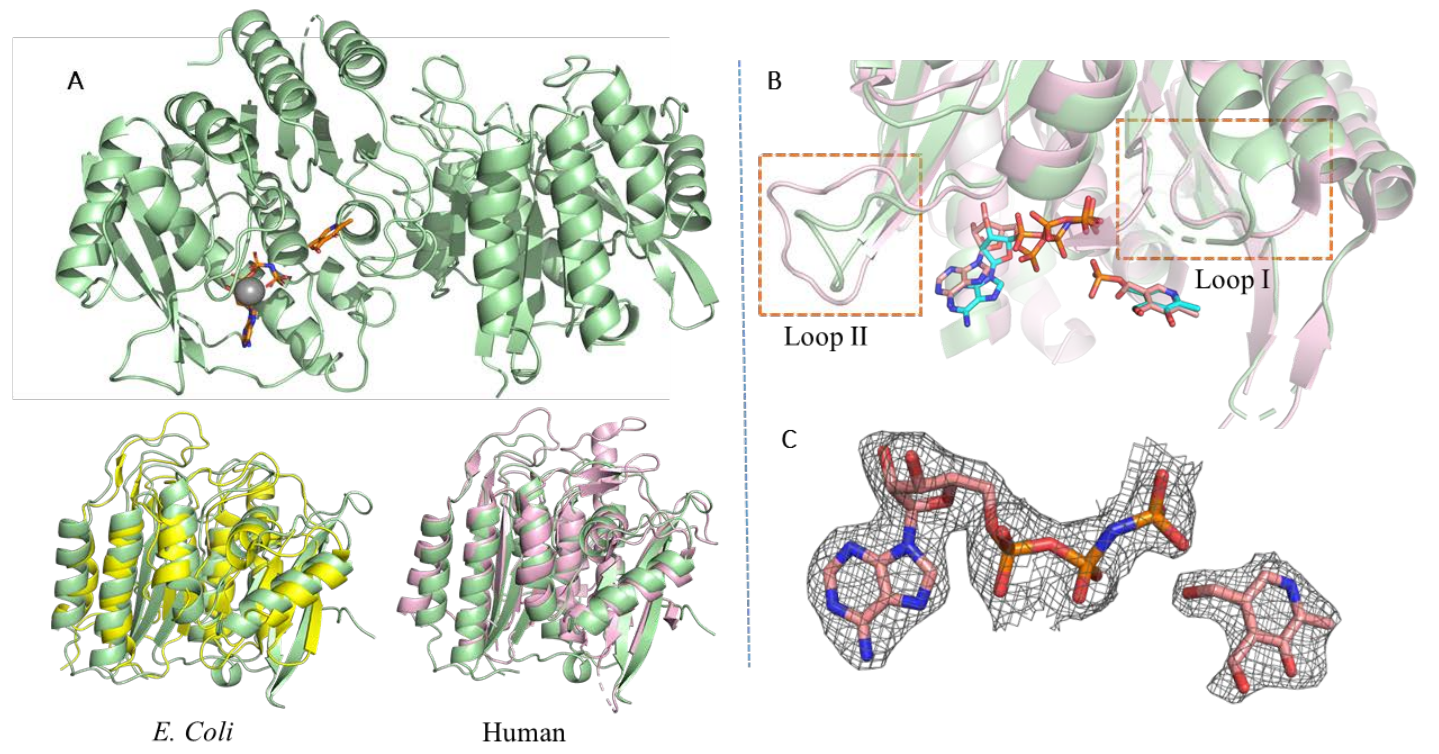

Figure 2. The structure of PfPdxK loaded with the non-hydrolysable ATP analog (AMP-PNP) and PL (green). (A) Structure alignment with PdxK from Human (pink) and E. coli (yellow), respectively, demonstrates the conserved nature of the fold; (B) an alignment of AMP-PNP and PL in PfPdxK (green) with the human complex (pink; 3KEU) with ADP and PLP shown in stick representation, showing the conserved nature of binding of both ATP and the substrate. (C) The 2Fo- $F c$ electron density map for AMP-PNP and PL (black mesh) within the PfPdxK structure (monomer A) contoured at $1 \sigma$. An omit map is for AMP-PNP and PL is shown in 3B and 3D.

\subsection{PfPdxK in Complex with AMP-PNP and PL}

The crystal structure of the PfPdxK complex was determined at $2.15 \AA$ (see Table 1 for crystallographic statistics) in space group P1211. Each asymmetric unit contains one molecule, and two monomers form a dimer by the two-fold crystallographic axis. Each monomer has an independent active site, with no contributions made from the opposing monomer. We found that the occupancy of AMP-PNP and PL is lower in monomer B than A, which is also visible in the higher B-factors of AMP-PNP and surrounding residues, indicating the PfPdxK can also undergo conformational changes in the absence/presence of substrate, as seen in other PdxK enzymes [6]. A characteristic feature in the ribokinase superfamily structure is the flap or lid that cover the substrate, as well as the ATP phosphates group. Among the three structures of PL kinases, the flap is defined by loop I and loop II. During the binding of AMP-PNP, the flexible loop I around the active site was reported to trigger a major conformational change of the protein structure [6]. However, rotation of the flexible loop II is responsible for making close contact with and stabilizing the substrates.

In PfPdxK, loop II adopts an extended conformation towards the buried active pocket and makes numerous interactions with PL. In one subunit, a conserved Cys18 on the flap binds to PL in a 
non-covalent fashion. Ser11, His48 and Asp429 form hydrogen-bonding interactions with PL. In PLKs, the flap is in a more open position, and a hydrophobic residue replaces the cysteine. The movement of the flap probably makes a major contribution to the binding affinity between different PL substrates and this variation may explain why the orientation of PL in the binding site of our structure is slightly different from that seen in others. In the PfPdxK complex, AMP-PNP and PL show a clear electron density signal in the active site $P f \mathrm{PdxK}$ (Figure 2C). The adenine base makes two hydrogen bonds to Ile420, while the phosphate groups form multiple hydrogen bonds with the protein backbone both directly or via solvents (Figure 3). All residues that interact with the phosphate groups are highly conserved within the ribokinase family. The $\mathrm{Mg}^{2+}$ ion is well coordinated by 4 highly ordered water molecules, Asp318 and the $\beta$ phosphate of AMP-PNP.

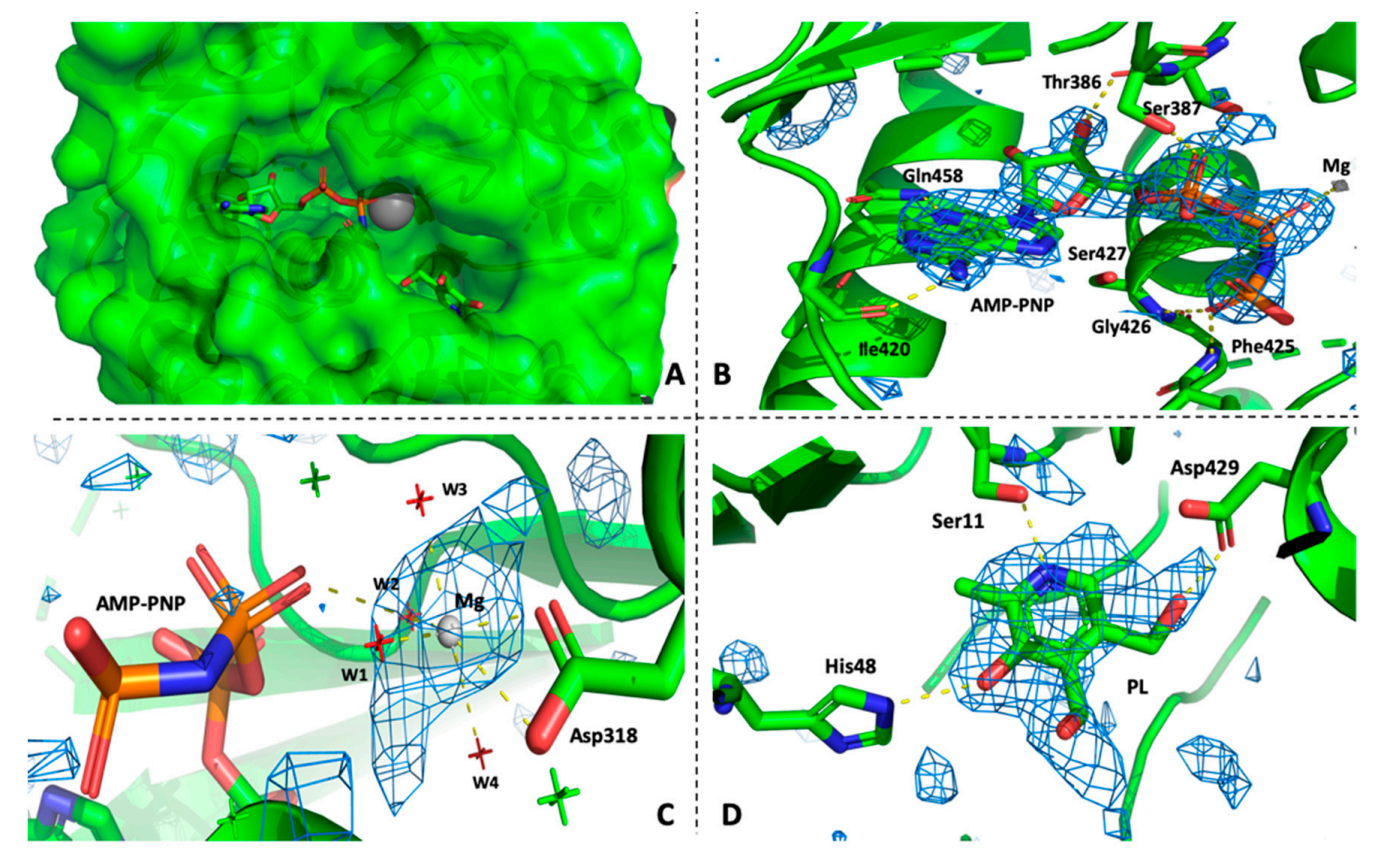

Figure 3. (A) Crystal structure of PfPdxK in complex with AMP-PNP, $\mathrm{Mg}^{2+}$ ion and PL, PfPdxK is shown in the surface representation and three ligands located deep inside the pocket; The Fo- $F c$ omit map of AMP-PNP (B), $\mathrm{Mg}^{2+}$ (C) and PL (D) within the PfPdxK structure. Hydrogen bonding interactions of AMP-PNP, PL and $\mathrm{Mg}^{2+}$ with PfPdxK are shown as yellow dashed lines, the four red labeled water around $\mathrm{Mg}^{2+}$ in (C) help to coordinate AMP-PNP and $\mathrm{Mg}^{2+}$ ion in the pockets.

\subsection{The Surface-Exposed Conserved FIxxIIxL Motif may Function as a Signal for Degradation}

In the structure, we found that the degenerate sequence (located between $\alpha 3$ to $\alpha 4$ from His 107 to Tyr303) was disordered. The equivalent region, connecting region $\alpha 3$ to $\alpha 4$, is a continuous loop or $\beta$ sheet in other PdxKs (Figure 4A). The degenerate sequence contains numerous MNXH or TNXH repeat motifs that have no structural homologues in eukaryotes (Figure 1). However, it seems to be a frequent phenomenon in many plasmodial proteins (Figure S1). Further examination indicated not only that MNXH or TNXH repeats were often present, but that another motif (FIxxIIxL) conserved across apicomplexa is also often present at the $\mathrm{C}$ terminus of the degenerate inserts in other apicomplexan genes (Figure S2). The FIxxIIxL motif occupies a distinct conformation to that seen in the linker between $\alpha 3$ and $\alpha 4$ of human PdxK, suggesting that it does not function as a replacement of the connection between helices $\alpha 3$ and $\alpha 4$ (Figure 4C). A similar motif has been described previously (the Engrailed Homology1 (Eh1) motif) which has the consensus sequence FxIxxIL, of which only F is completely conserved. This motif functions by allowing the recruitment of other proteins. For example, the Eh1 motif was found to interact with the WD40 repeat domains of the Groucho/TLE co-repressor [25] (2CE8). Our model demonstrates not only that the FIxxIIxL motif is present as a surface-exposed epitope, but that it also adopts a similar conformation to that previously seen in the interaction of Eh1 with 
WD40 repeats [25] (Figure 4A). The WD40 repeat consists of 40 amino acids, terminated with Trp-Asp (W-D) dipeptides. Repeated WD40 motifs act as a site for protein-protein interaction, and proteins containing WD40 repeats are known to serve as platform for the assembly of protein complexes, or mediators of temporal control among other proteins like $G$ proteins, the TAFII transcription factor, or E3 ubiquitin ligase [26,27]. WD40 repeats usually assume a 7-8 bladed $\beta$-propeller fold. Proteins have been found with 4 to 16 repeated units, which also form a circular $\beta$-propeller structure [28]. In this structure, the Eh1 peptide binds over the mouth of the central channel of the TLE-WD40 domain (Figure 4B) and the central core of the Eh1 motif (from Ser2 to Leu7) forms a short amphipathic helix, following a very similar conformation to the FIxxIIxL motif in PfPdxK.

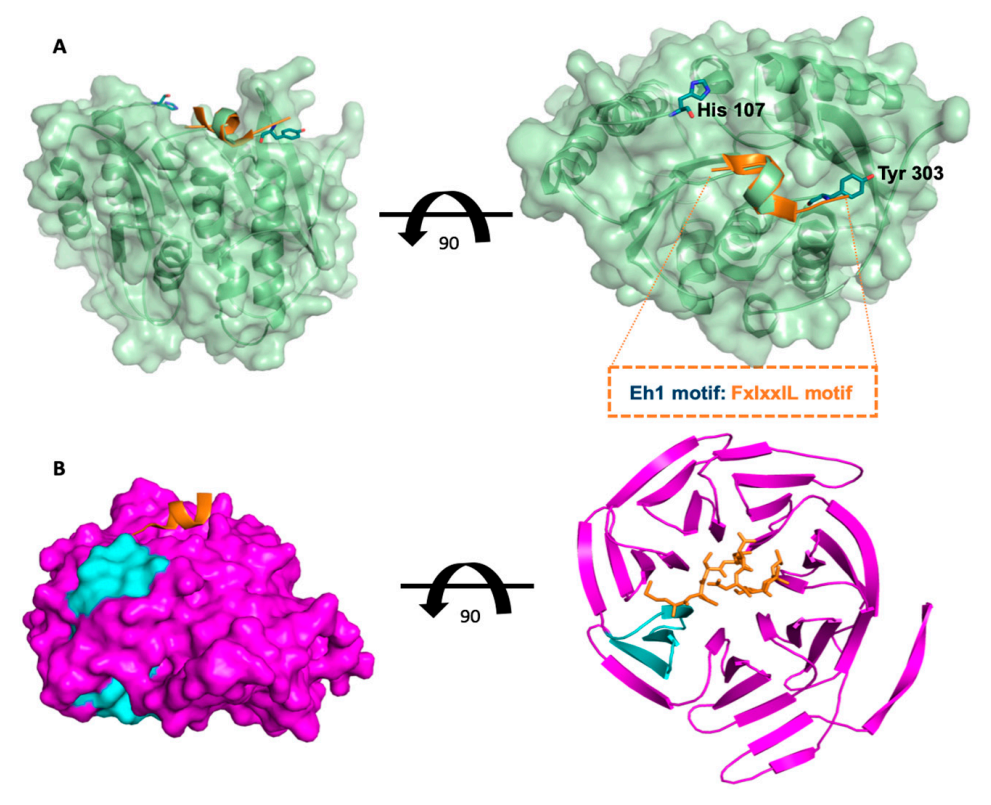

C

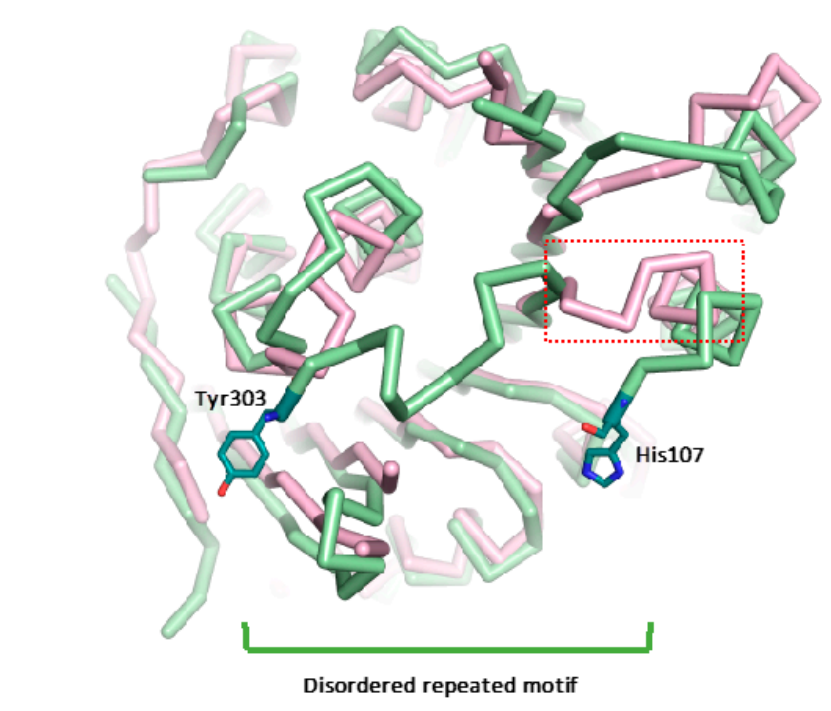

\begin{tabular}{|l|l|l}
\hline MNXH & TNXH & MNXH \\
\hline
\end{tabular}

Figure 4. A surface-exposed epitope in PfPdxK resembles the known Engrailed Homology1 (Eh1) motif. (A) View of an apicomplexian-conserved motif FIxxIIxL located on the surface of PfPdxK, $P f \mathrm{PdxK}$ is shown in green and an overlap of the Eh1 peptide is shown in orange. (B) Representation of the Eh1 motif (FxIxxIL) bound to the Groucho/TLE co-repressor with the WD40 domain shown in cyan. (C) Structure alignment of Human (pink) and PfPdxK (green), demonstrating that the Eh1-like epitope does not directly replace the function of linking $\alpha 3$ to $\alpha 4$. 


\subsection{Molecular Docking Indicates Potential Binding Modes of PT3/PHME/PT5 as Substrates of PfPdxK}

The novel non-phosphorylated pyridoxyl-adducts compounds PT3, PT5 and PHME were tested in an anti-Plasmodial assay, with results showing that PT3 can inhibit the proliferation of P. falciparum with $\mathrm{IC}_{50}$ of $14 \mu \mathrm{M}$ [15]. Pf PdxK was strongly implicated in the phosphorylation of PT3, PT5 and PHME into their active forms. As these three pro-drugs possessed distinct effects on Plasmodial cultures, we used our model to attempt to explain the differences in effect, based on their suitability for phosphorylation by $P f \mathrm{PdxK}$. To assess whether they were potential substrates for $P f \mathrm{PdxK}$ and predict their binding models, we utilized the Swissdock online server based on grid dock to model the PT3/PHME/PT5 binding modes, respectively. As ATP is the source for PL phosphorylation, we prepared a single-molecule PfPdxK with AMP-PNP bound as our molecular target protein, and non-phosphorylated pyridoxal-adducts PT3/PHME/PT5 as ligands to perform rigid docking (repeated three times independently).

According to a phosphorylation assay in vivo, PT3/PHME/PT5 were confirmed to be phosphorylated by PfPdxK. All three were phosphorylated by the Plasmodial enzyme with a specific activity of 38, 20 and $64 \mathrm{nmol} \mathrm{min}{ }^{-1} \mathrm{~m}^{-1}$, respectively [15]. We ranked potential docking on the lowest energy, selecting for positions immediately proximal to the AMP-PNP binding pocket that also possessed strong overlaps with the conserved PL-moiety (Figure 5). Our modeling suggests that the pyridoxal group of PHME and PT5 fit well onto the known PfPdxK binding mode, but that the pyridoxal group of PT3 presented a potential steric clash with AMP-PNP, which might weaken the interaction of PT3 under the same conditions or require rearrangement of the ATP binding mode for phosphorylation to proceed. This calculation is consistent with the experiments that demonstrate PHME and PT5 possess better activities than PT3 as substrates of PfPdxK and may provide the basis for further development of this pro-drug class.

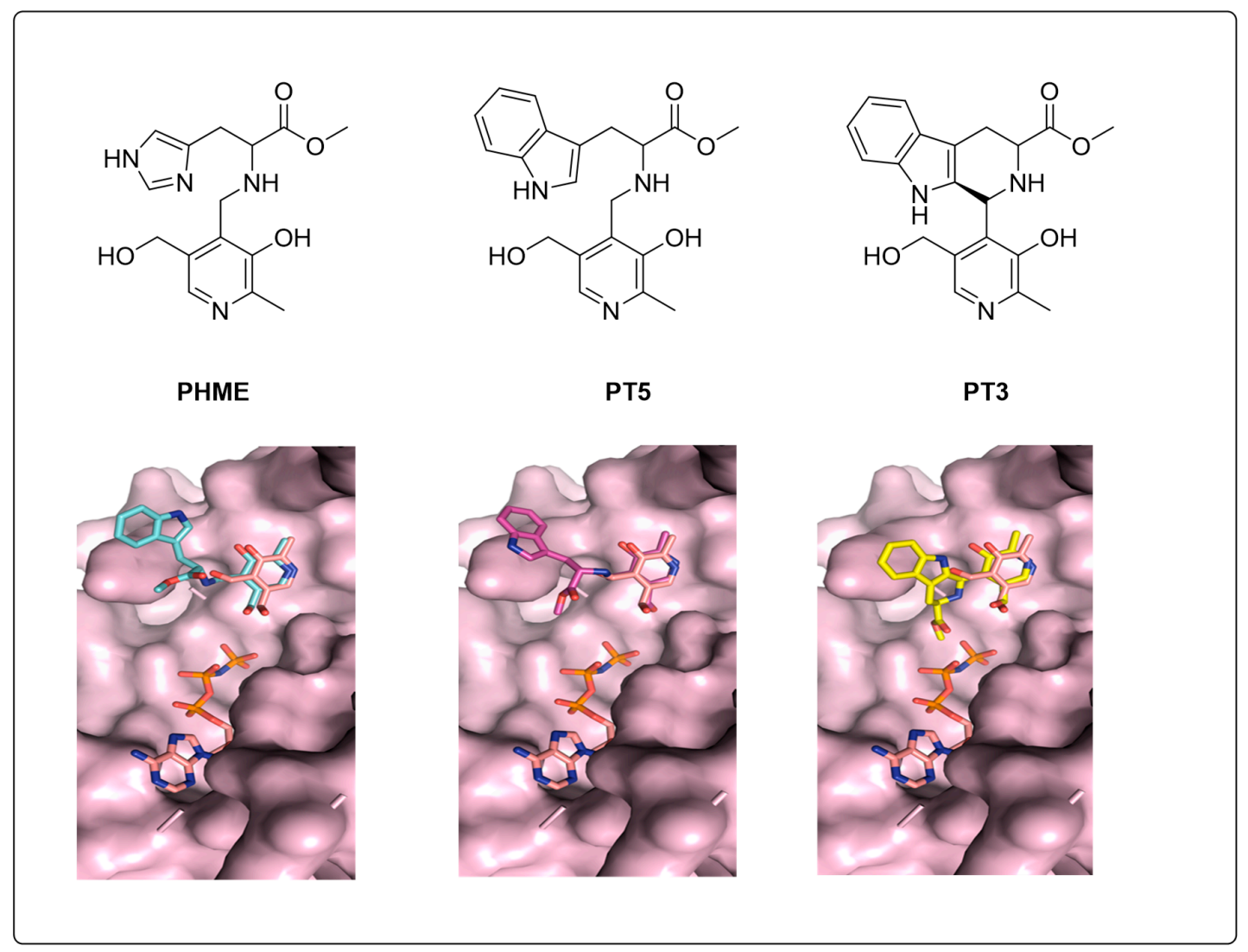

Figure 5. Molecular docking of PT3/PHME/ to PfPdxK overlapped with AMP-PNP and PL. 


\section{Discussion}

Based on the crystal diffraction data we obtained with AMP-PNP and PL, the overall structure of $P f \mathrm{PdxK}$ shares a similar conformation with human and E. coli PdxK. The binding pocket for multiple substrates and ATP are conserved among the species as alignments indicated within the three species. Unfortunately, we were unable to detect any electron density signal from the repeating motif region, likely due to degradation during crystal growth. While the lack of a stable structure result is expected, we cannot exclude the possibility that this repeat region may become structured upon binding to a third, as yet unknown, partner. Similar repeats are found in other malarial sp. (Figure S1), suggesting a commonality of role for these repeats. As this repeat region is located at the opposite side of the protein to the substrate-binding pocket, it is unlikely that the motif itself would affect $P f P d x K$ function as a kinase. An examination of the solvent content of the crystal indicates that the truncated form of $P f \mathrm{PdxK}$ presented here would possess a solvent content of $58 \%$, in line with the solvent content often found in crystals, whereas the full-length $P f \mathrm{PdxK}$ would possess a solvent content of $8 \%$. Such a low value is extremely unlikely, suggesting that either a minor contaminant or auto-proteolysis is responsible for the removal of this degenerate sequence during the crystallisation process.

A potential explanation for this presence of the degenerate sequence in the wild-type enzyme is that these repeats provide a signal for post-translational control of $P f \mathrm{PdxK}$ through an interaction with other proteins. Based upon a similarity between the FIxxIIxL motif found in PfPdxK and the FxIxxIL motif found in the Engrailed Homology1 (Eh1) motif (both in sequence and in structure), we propose that one mechanism could be through the recruitment of a WD40 domain protein, that in turn, may recruit a E3 ligase to drive ubiquitin-mediated degradation of $P f \mathrm{PdxK}$ and other plasmodial proteins containing similar pairings of degenerate MNXH or TNXK repeats with a C-terminal FIxxIIxL motif. This hypothesis is based on the report that the Eh1 motif may recruit WD40 proteins [29]. However, we have no data to support a direct interaction between a WD40 domain protein and $P f \mathrm{PdxK}$. Why the parasite, and other apicomplexians, require fine control of proteins, such as PfPdxK, post-translationally remains an interesting open question.

While our model provides a molecular snapshot of ATP and PL binding to a pyridoxal kinase it should be borne in mind that interpretation of the electron density is always subjective. Our omit maps do indicate that PL is bound in the manner described, but we cannot exclude the possibility that some of the PfPdxK molecules within the lattice do not have a bound PL, but are either empty or have a bound glycerol (present in the cryo-buffers used). However, our deposited model has good agreement in terms of PL orientation with other PL-bound pyridoxal kinases and the modeling of PT3, PT5 and PHME also suggest this is a binding site for PL-like molecules.

Additionally, we generated models by molecular docking to explain how PfPdxK phosphorylates PT3, PT5 and PHME into the potential active anti-malaria drug. Our analysis shows that the predicted mode of binding of these molecules effectively explains the observed differences in their effect, with the differences in molecular properties in vivo accounted for by potential steric clashes with residues lining the binding pocket. However, it should be borne in mind that these results are obtained from in silico modeling and confirmation of the binding mode awaits experimental validation. The structure of the wild-type Plasmodium PdxK provides further insight into the molecular basis of active PT3/PHME/PT5 as pro-drugs. In all, the structure of $P f \mathrm{PdxK}$ provides further basis for the understanding of P. falciparum parasite vitamin salvage.

Supplementary Materials: The following are available online at http://www.mdpi.com/2073-4352/9/10/534/s1, Figure S1: BLAST results of the MNXH or TNXH repeats domain alignment, commonly shown in plasmodium genome, Figure S2: Alignment of different resource plasmodial falciparum contain a common FIxxIIxL motif after the $\mathrm{xNxH}$ repeat motif domains, shows in the red square.

Author Contributions: Conceptualization, M.R.G. and C.W.; validation and analysis, K.G., W.W. and T.K.; data curation, K.G., W.W. and M.R.G.; writing-original draft preparation, all authors; writing-review and editing, all authors; visualization, K.G. and T.K.; supervision and project administration M.R.G.; funding acquisition, M.R.G. and C.W. 
Funding: This research was funded by CSC Fellowships to KG and WW. The authors would like to also acknowledge the Fundação de Amparo à Pesquisa do Estado de São Paulo (FAPESP, grants 2014/03644-9 to TK, 2017/03966-4 and 2015/26722-8 to CW). This project has received funding from the European Union's Framework Program for Research and Innovation Horizon 2020 (2014-2020) under the Marie Skłodowska-Curie Grant Agreement No. 675555, Accelerated Early staGe drug discovery (AEGIS).

Acknowledgments: The authors would like to acknowledge access to synchrotron radiation at beamline X11, EMBL Hamburg and P11, PETRA III, DESY, Hamburg.

Conflicts of Interest: The authors declare no conflict of interest. The funders had no role in the design of the study; in the collection, analyses, or interpretation of data; in the writing of the manuscript, or in the decision to publish the results.

\section{References}

1. Percudani, R.; Peracchi, A. A genomic overview of pyridoxal-phosphate-dependent enzymes. EMBO Rep. 2003, 4, 850-854. [CrossRef] [PubMed]

2. Sivaraman, J.; Li, Y.; Banks, J.; Cane, D.E.; Matte, A.; Cygler, M. Crystal Structure of Escherichia Coli PdxA, an Enzyme Involved in the Pyridoxal Phosphate Biosynthesis Pathway. J. Biol. Chem. 2003, 278, 43682-43690. [CrossRef] [PubMed]

3. Müller, I.B.; Hyde, J.E.; Wrenger, C. Vitamin B Metabolism in Plasmodium falciparum as a Source of Drug Targets. Trends Parasitol. 2010, 26, 35-43. [CrossRef] [PubMed]

4. Yang, Y.; Zhao, G.; Winkler, M.E. Identification of the Pdxk Gene That Encodes Pyridoxine (Vitamin B6) Kinase in Escherichia Coli K-12. FEMS Microbiol. Lett. 1996, 141, 89-95. [CrossRef] [PubMed]

5. Yang, Y.; Tsui, H.C.; Man, T.K.; Winkler, M.E. Identification and Function of the Pdxy Gene, Which Encodes a Novel Pyridoxal Kinase Involved in the Salvage Pathway of Pyridoxal 5'-Phosphate Biosynthesis in Escherichia Coli K-12. J. Bacteriol. 1998, 180, 1814-1821.

6. Li, M.H.; Kwok, F.; Chang, W.R.; Liu, S.Q.; Lo, S.C.; Zhang, J.P.; Jiang, T.; Liang, D.C. Conformational Changes in the Reaction of Pyridoxal Kinase. J. Biol. Chem. 2004, 279, 17459-17465. [CrossRef]

7. Cao, P.; Gong, Y.; Tang, L.; Leung, Y.C.; Jiang, T. Crystal Structure of Human Pyridoxal Kinase. J. Struct. Biol. 2006, 154, 327-332. [CrossRef]

8. Safo, M.K.; Musayev, F.N.; di Salvo, M.L.; Hunt, S.; Claude, J.B.; Schirch, V. Crystal Structure of Pyridoxal Kinase from the Escherichia Coli Pdxk Gene: Implications for the Classification of Pyridoxal Kinases. J. Bacteriol. 2006, 188, 4542-4552. [CrossRef]

9. Newman, J.A.; Das, S.K.; Sedelnikova, S.E.; Rice, D.W. The crystal structure of an ADP complex of Bacillus subtilis pyridoxal kinase provides evidence for the parallel emergence of enzyme activity during evolution. J. Mol. Biol. 2006, 363, 520-530. [CrossRef]

10. Bishop, O.T.; Wells, G.A.; Joubert, F.; Wrenger, C.; Walter, R.D.; Louw, A.I. Progress Towards Determining the Structure of Plasmodium falciparum Pyridoxal Kinase. In Proceedings of the First Southern African Bioinformatics Workshop, Johannesburg, South Africa, 28-30 January 2007; pp. 41-44.

11. Kronenberger, T.; Lunev, S.; Wrenger, C.; Groves, M.R. Purification, Crystallization and Preliminary X-Ray Diffraction Analysis of Pyridoxal Kinase from Plasmodium falciparum (PfPdxK). Acta Crystallogr. Sect. F Struct. Biol. Cryst. Commun. 2014, 70, 1550-1555. [CrossRef]

12. Wrenger, C.; Eschbach, M.L.; Müller, I.B.; Warnecke, D.; Walter, R.D. Analysis of the Vitamin B6 Biosynthesis Pathway in the Human Malaria Parasite Plasmodium falciparum. J. Biol. Chem. 2005, 280, 5242-5248. [CrossRef] [PubMed]

13. Reeksting, S.B.; Muller, I.B.; Burger, P.B.; Burgos, E.S.; Salmon, L.; Louw, A.I.; Birkholtz, L.M.; Wrenger, C. Exploring Inhibition of Pdx1, A Component of the PLP Synthase Complex of the Human Malaria Parasite Plasmodium falciparum. Biochem. J. 2013, 449, 175-187. [CrossRef] [PubMed]

14. Muller, I.B.; Bergmann, B.; Groves, M.R.; Couto, I.; Amaral, L.; Begley, T.P.; Walter, R.D.; Wrenger, C. The Vitamin B1 Metabolism of Staphylococcus aureus Is Controlled at Enzymatic and Transcriptional Levels. PLoS ONE 2009, 4, e7656. [CrossRef] [PubMed]

15. Müller, I.B.; Wu, F.; Bergmann, B.; Knöckel, J.; Walter, R.D.; Gehring, H.; Wrenger, C. Poisoning Pyridoxal 5-Phosphate-Dependent Enzymes: A New Strategy to Target the Malaria Parasite Plasmodium falciparum. PLoS ONE 2009, 4, e4406. [CrossRef] [PubMed] 
16. Gardner, M.J.; Hall, N.; Fung, E.; White, O.; Berriman, M.; Hyman, R.W.; Paulsen, I.T.; Carlton, J.M.; Pain, A.; Nelson, K.E.; et al. Genome sequence of the human malaria parasite Plasmodium falciparum. Nature 2002, 419, 498-511. [CrossRef]

17. Kabsch, W. Xds. Acta Crystallogr. Sect. D Biol. Crystallogr. 2010, 66, 125-132. [CrossRef]

18. Evans, P.R.; Murshudov, G.N. How good are my data and what is the resolution? Acta Crystallogr. Sect. D Biol. Crystallogr. 2013, 69, 1204-1214. [CrossRef]

19. McCoy, A.J.; Grosse-Kunstleve, R.W.; Adams, P.D.; Winn, M.D.; Storoni, L.C.; Read, R.J. Phaser crystallographic software. J. Appl. Cryst. 2007, 40, 658-674. [CrossRef]

20. Emsley, P.; Cowtan, K. Coot: Model-Building Tools for Molecular Graphics. Acta Crystallogr. Sect. D Biol. Crystallogr. 2004, 60, 2126-2132. [CrossRef]

21. Murshudov, G.N.; Skubák, P.; Lebedev, A.A.; Pannu, N.S.; Steiner, R.A.; Nicholls, R.A.; Winn, M.D.; Long, F.; Vagin, A.A. Refmac5 for the Refinement of Macromolecular Crystal Structures. Acta Crystallogr. Sect. D Biol. Crystallogr. 2011, 67, 355-367. [CrossRef]

22. Grosdidier, A.; Zoete, V.; Michielin, O. Swissdock, a Protein-Small Molecule Docking Web Service Based on Eadock Dss. Nucleic Acids Res. 2011, 39, W270-W277. [CrossRef] [PubMed]

23. Notredame, C.; Higgins, D.G.; Heringa, J. T-Coffee: A Novel Method for Fast and Accurate Multiple Sequence Alignment. J. Mol. Biol. 2000, 302, 205-217. [CrossRef] [PubMed]

24. Robert, X.; Gouet, P. Deciphering Key Features in Protein Structures with the New Endscript Server. Nucleic Acids Res. 2014, 42, W320-W324. [CrossRef] [PubMed]

25. Jennings, B.H.; Pickles, L.M.; Wainwright, S.M.; Roe, S.M.; Pearl, L.H.; Ish-Horowicz, D. Molecular Recognition of Transcriptional Repressor Motifs by the WD Domain of the Groucho/Tle Corepressor. Mol. Cell 2006, 22, 645-655. [CrossRef] [PubMed]

26. Li, D.; Roberts, R. WD-Repeat Proteins: Structure Characteristics, Biological Function, and Their Involvement in Human Diseases. Cell. Mol. Life Sci. 2001, 58, 2085-2097. [CrossRef]

27. Neer, E.J.; Schmidt, C.J.; Nambudripad, R.; Smith, T.F. The Ancient Regulatory-Protein Family of WD-Repeat Proteins. Nature 1994, 371, 297-300. [CrossRef]

28. Xu, C.; Min, J. Structure and Function of WD40 Domain Proteins. Protein Cell 2011, 2, 202-214. [CrossRef]

29. Copley, R.R. The EH1 motif in metazoan transcription factors. BMC Genom. 2005, 6, 169-177. [CrossRef] 